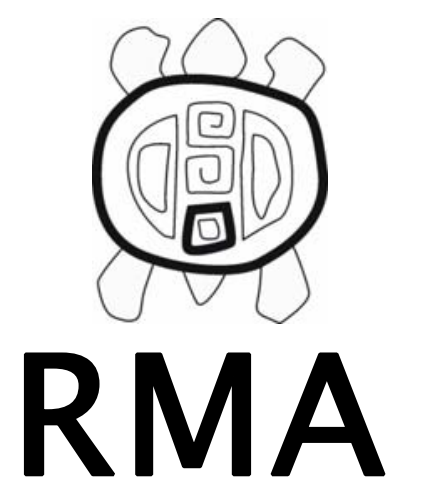

Antropología Biológica

\title{
Patrones geográficos de diferenciación craneofacial entre poblaciones de la región central del país y otras de Argentina: aportes desde la genética del paisaje
}

\author{
Mariana Fabra \\ Becaria de Postgrado CONICET/Museo de Antropología \\ Facultad de Filosofía y Humanidades \\ Universidad Nacional de Córdoba. \\ mfabra@ffyh.unc.edu.ar
}

Resumen

En este trabajo se analizan los patrones de variación espacial de los grupos humanos que habitaron la región central del país (actualmente, parte de los territorios de las provincias de Córdoba y San Luis) conocida en la arqueología como «Sierras Centrales», y otras regiones ecológicas de la Argentina, buscando identificar barreras de diferenciación morfológica craneofacial en un amplio espacio geográfico. El estudio se basa en el análisis de 10 variables morfométricas lineales en 14 muestras representativas de poblaciones que habitaron diversas regiones del país, y se aplica análisis de componentes principales -PCA-para el estudio de las relaciones biológicas entre poblaciones, y el algoritmo de máxima diferenciación de Monmonier-AM- para la identificación de barreras genéticas. Los resultados obtenidos a partir de PCA y AM son coincidentes, y permiten señalar que las poblaciones asentadas en la región central del país presentan mayores similitudes morfológicas con poblaciones de la Patagonia y del Noreste de la región pampeana, más que con grupos asentados en el Noroeste, Noreste y Centro-Oeste del país. Estos resultados apoyan nuestra hipótesis acerca de un poblamiento de esta región procedente del Noreste del país, que luego habría continuado hacia Patagonia y Tierra del Fuego.

Palabras clave: Sudamérica, poblamiento, variables lineales, estructura genética, barreras genéticas, algoritmo de máxima diferenciación de Monmonier.

Geographical patterns of craniofacial variation among populations from Central Argentina and surrounding areas. A landscape genetics contribution.

\begin{abstract}
In this study we analyze the patterns of spatial variation of a population sample from the pre-Hispanic inhabitants of the central territory of Argentina (Córdoba and San Luis provinces) archaeologically known as «Sierras Centrales», and 13 other samples from different ecological and geographical regions from Argentina. Additionally, we searched for the existence of genetic barriers in the geographic landscape. The study is based on 10 craniometric measurements. Results from principal component analysis reveal that the pre-Hispanic inhabitants of the Central Mountains of Argentina were biologically more related to the Patagonian groups than to the northeast, central western and northwestern populations. Monmonier 's algorithm reveals that the pre-Hispanic inhabitants of the Central Mountains of Argentina were biologically more related to the Patagonian groups than to the central and northern populations. These results support our hypothesis that the peopling of the central region of Argentina most likely took place as a migratory wave proceeding from the northeast, and continued southward to Patagonia and Tierra del Fuego.
\end{abstract}

Keywords: South America, peopling, craniometrics, genetic structure, genetic barriers, Monmonier's algorithm.

\section{Introducción}

Generalmente, cuando se establecen similitudes biológicas entre poblaciones, una pregunta que usualmente queda sin responder es la de los límites o barreras que configuran dicho espacio genético. Los diferentes métodos estadísticos de ordenación (análisis de componentes principales, análisis de coordenadas principales, análisis de escalamiento multidimensional) permiten identificar las afinidades genéticas entre poblaciones, pero no los límites geográficos de la variabilidad observada. En este trabajo, nos interesa investigar las zonas o regiones de cambio significativo en el flujo génico de poblaciones asentadas en diversos espacios geográficos de la Argentina, zonas que pueden estar asociadas con barreras 
geográficas o culturales, desde la perspectiva de la genética del paisaje (ver revisión histórica y de técnicas en Manel et al. 2003). Esta perspectiva de reciente desarrollo surge de la combinación de la genética de poblaciones y la ecología del paisaje, y permite una mayor comprensión de la interacción entre el paisaje y los procesos microevolutivos tales como flujo génico, deriva génica y selección. Además, posibilitaría la identificación de barreras, las que pueden ser definidas como aquellas zonas donde las diferencias entre pares de poblaciones son mayores, produciendo un brusco cambio en la distancia biológica de las mismas.

Con respecto a la variabilidad biológica de las poblaciones originarias de la Argentina, si bien han sido extensamente estudiadas ${ }^{1}$ a partir de análisis morfométricos lineales (Barrientos et al. 2003; Cocilovo et al. 1999; Cocilovo y Di Rienzo 1984-1985; Cocilovo y Neves 1988-1989; González-José 2003; GonzálezJosé et al. 2001 a, 2001 b; Hernández et al. 1997; Lahr 1995; Lalueza et al. 1996, 1997; Marcellino y Colantonio 2000; Mendez y Salceda 1995; Rothammer et al. 1984, 1988- 1989; Sardi et al. 2005) y más recientemente, mediante análisis de morfometría geométrica (Bernal et al. 2006; González-José et al. 2003; Pérez 2006; Pérez et al. 2007) y estudios moleculares (Cabana et al. 2006; Dejean et al. 2004; Dipierri et al. 1998, 2000; Demarchi 2000; Demarchi et al. 2001, 2005; Goicoechea et al. 2001), numerosas preguntas acerca de los orígenes, las estrategias de dispersión así como la posterior evolución local de los grupos colonizadores permanecen no resueltas. Con relación al estado actual de conocimiento sobre las poblaciones prehispánicas del área central del territorio argentino, existen dos grandes temas aún por resolver y que también son significativos en un contexto más amplio, el de la evolución de las poblaciones americanas. Uno es el poblamiento inicial y el proceso posterior de colonización y expansión en este espacio; otro se refiere a la evolución biológica de esas poblaciones, es decir, la importancia relativa que tuvieron sobre ellas las fuerzas evolutivas direccionales (migración, selección) y las aleatorias (efecto fundador, deriva genética). Estudios anteriores basados en medidas lineales sobre muestras osteológicas de la región austral de las Sierras Pampeanas han propuesto diversas y a veces contrapuestas hipótesis para explicar el poblamiento de la región. Basados en la evidencia arqueológica, y en menor medida, antropológica, se ha asociado estas poblaciones con corrientes migratorias procedentes del Noroeste de nuestro país (Cocilovo 1984; González 1944; Serrano 1945; Outes 1911). Otros autores señalaron semejanzas morfológicas con poblaciones de la region cuyana, en el centro-oeste del país (Canals Frau 1944). Más recientemente, otros investigadores han verificado, a través de análisis morfométricos lineales, similitudes entre los habitantes de la región central del país y los

Se mencionan solamente algunos de los trabajos sobre el tema, no pretende ser una revisión exhaustiva de la bibliografía disponible. pueblos que ocuparon Cuyo y el Sur del Litoral (Cocilovo 1984; Marcellino y Colantonio 1993). Nuestros propios resultados obtenidos a partir de marcadores del ADN mitocondrial (García y Demarchi 2006), rasgos epigenéticos y variables métricas lineales (Demarchi et al. 2007; Fabra etal. 2005, 2007; Fabra 2008a, 2008b; Laguens et al. 2007a), y evidencia arqueológica (Laguens et al. 2007b, 2007c) abonan la hipótesis de un origen común para las poblaciones que ocuparon la región central del país con los grupos humanos que habitaron Patagonia y Tierra del Fuego.

En este marco, aplicando una metodología que maximiza la diferencia genética entre poblaciones algoritmo de máxima diferencia de Monmonier (1973)el objetivo del presente estudio se centra en analizar los patrones espaciales de variabilidad morfológica craneofacial de poblaciones humanas que habitaron diversas regiones geográfico-culturales de la Argentina durante el Holoceno tardío, particularmente con relación al poblamiento de la región central del país, esperando identificar barreras de diferenciación genética en un vasto paisaje geográfico.

\section{Materiales y Métodos}

\section{La muestra}

La muestra de la región central del país está compuesta por 64 adultos masculinos procedentes de 23 sitios arqueológicos de la provincia de Córdoba (CBA) y 9 cráneos de diversa procedencia arqueológica en la provincia de San Luis (SL); estos últimos materiales carecen de cronologías absolutas, aunque se supone corresponden al Holoceno tardío (ver Tabla 1 y Figura 1).

Esta región ocupa parte del extremo austral de las Sierras Pampeanas, entre los $30-33^{\circ}$ grados de latitud Sur y $62-65^{\circ}$ grados de longitud Oeste (Figura 1). La fisiografía de esta región presenta contrastes entre la región serrana y la llanura. Las tres cadenas montañosas de las sierras -Sierras Grandes, Sierras Chicas, Sierras Occidentales- se ven intercaladas por valles intermontanos longitudinales, quebradas y pampas. Al Oeste, Este y Sur de las sierras se encuentran las planicies. De acuerdo a su ubicación con respecto al sistema serrano se las denomina Ilanura oriental -de características similares a la llanura pampeana en su parte media inferior, y chaco-pampeana en su parte septentrional- y occidental. Entre las sierras y la llanura, el piedemonte, con una altura sobre el nivel del mar que no supera los $600 \mathrm{~m}$ (Capitanelli 1979).

La evidencia arqueológica señala que en esta región se desarrollaron diversas estrategias y adaptaciones al ambiente. Progresivamente, a partir de 8000 A.P., se observa la ocupación de nuevos espacios y el mayor aprovechamiento de los ya colonizados, la aparición de nuevas tecnologías y modalidades de explotación de recursos. La posterior adopción de la agricultura y la alfarería -aproximadamente 1500 A.P.-, y los 
consecuentes desarrollos regionales le otorgan a estas poblaciones características de unicidad que las distinguen de lo observado en otras regiones del país. Estas características distintivas habrían llevado a los conquistadores a denominar de una manera homogeneizante a los habitantes de la región como «Comechingones». Sin embargo, las diferentes estrategias y desarrollos locales observados llevan a pensar en una diversidad de grupos étnicos que contradicen la supuesta unicidad cultural de la región, postulada por los primeros cronistas (Laguens 1999; Laguens y Bonnin 2000).

Se poseen fechados radiocarbónicos mediante AMS para 6 de las muestras incluidas en este estudio (Tabla 2), que las ubican a fines del Holoceno medio y Holoceno tardío. Para el resto de las muestras, se posee en algunos casos información sobre las características de la inhumación o contextos arqueológicos asociados, que permiten suponer una cronología relativa para el Holoceno tardío (2500-400 A.P.).

Para los análisis interpoblacionales se incorporaron 169 adultos masculinos de 12 poblaciones que ocuparon durante el Holoceno tardío diversos ambientes y desarrollaron variadas estrategias adaptativas (ver Tabla 1 y Figura 2). Las muestras del Noreste de la Patagonia proceden del Valle del Río Negro (RNV), de la localidad de San Antonio Oeste (SAO) y de la Península San Blas e Isla Gama (SBI). Estas muestras

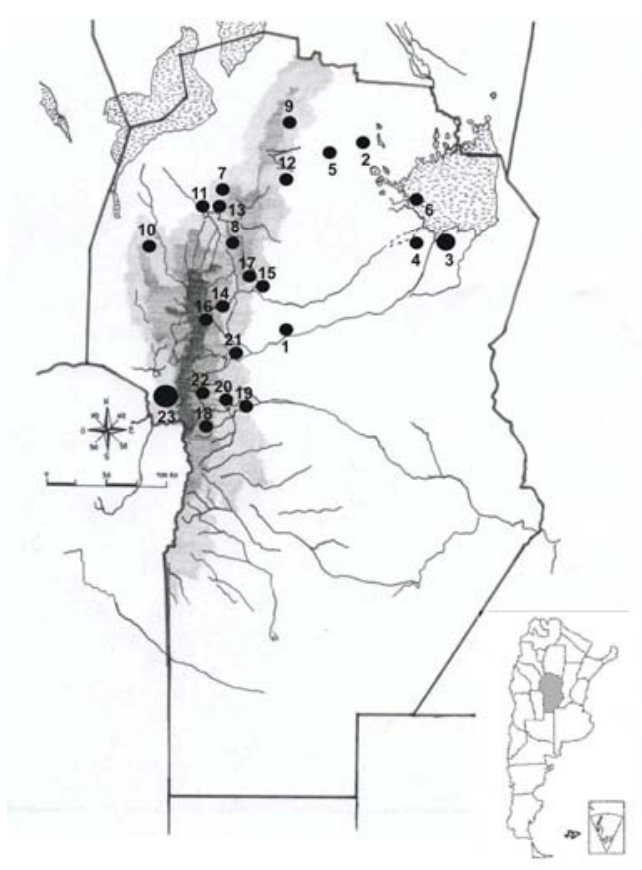

Figura 1. Mapa de la región austral de las Sierras Pampeanas señalando la ubicación de los 23 sitios arqueológicos incluidos en este análisis: 1) Cosme / Rincón, 2) Laguna de la Sal, 3) Miramar, 4) La Para, 5) Pozo de las Ollas, 6) Isla Tigre, 7) Nunsacate, 8) Ayampitin, 9) Cerro Colorado, 10) Charquina, 11) Cruz del Eje, 12) Guayascate, 13) Ischilin, 14) Cuesta Blanca, 15) Parque Sarmiento, 16) Copina, 17) Unquillo, 18) Amboy, 19) Río Tercero), 20) Villa Rumipal, $21)$ Potrero de Garay, 22) Yacanto, 23) Traslasierra. El mayor diámetro para las referencias 3 y 23 denota la utilización de varias muestras arqueológicas.

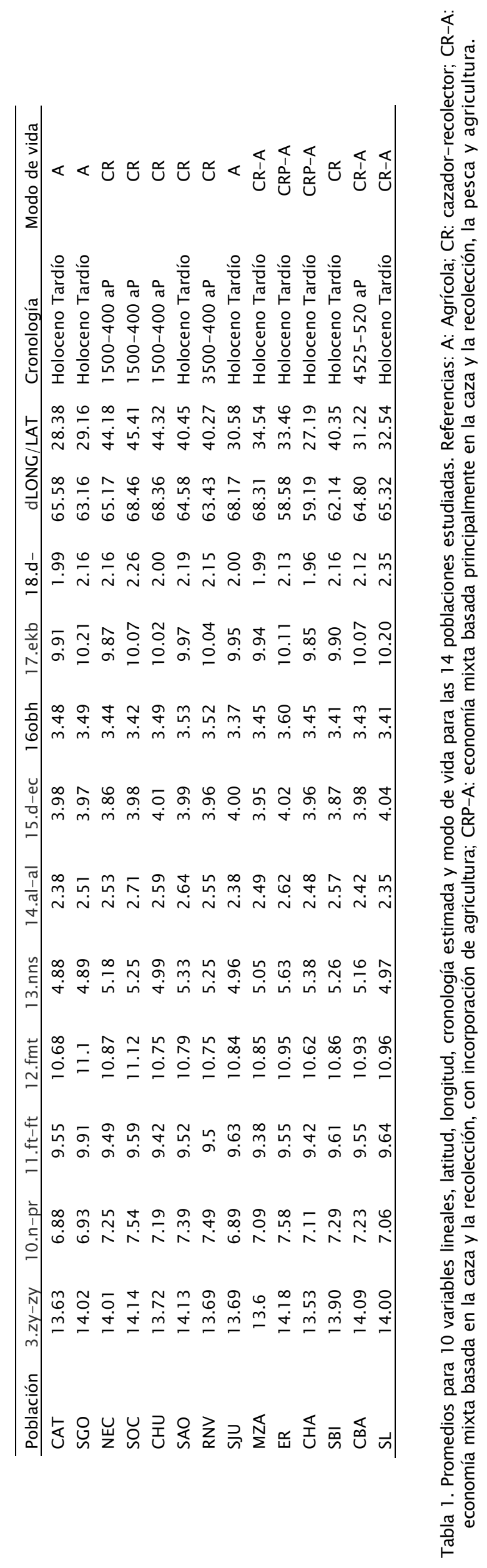




\begin{tabular}{lcccc}
\hline Muestra & Años C14 AP & Código UCIAMS & Deformación & Cronología \\
\hline Mir5 ZS2 & $4525 \pm 20$ & 22288 & - & HM \\
LP1 & $3810 \pm 20$ & 39099 & PC & HM \\
CE LP I1 & $3805 \pm 20$ & 22284 & PC & HM \\
G98 I2 & $920 \pm 20$ & 22281 & - & HTaf \\
Amboy I2 & $830 \pm 20$ & 22283 & - & HTaf \\
Ayampl1 & $600 \pm 20$ & 22287 & - & HTaf \\
\hline
\end{tabular}

Tabla 2. Fechados radiocarbónicos por AMS para muestras osteológicas de la región austral de las Sierras Pampeanas incluidas en el presente estudio. Referencias: Código UCIAMS: Código Universidad de California, Irving; Def. (Deformación): PC: deformación pseudo-circular; Muestras: Mir5 ZS2: sitio Miramar, individuo 5; LP1: sitio La Para, individuo 1; CE LP I1: sitio Costa Este Laguna del Plata, individuo 1; G98 12: sitio Guasmara, individuo 2; Amboy 12: sitio Amboy, individuo 2; Ayamp 11: sitio Ayampitin, individuo 1; Cronología: HM: Holoceno medio; HTf: Holoceno tardío final.

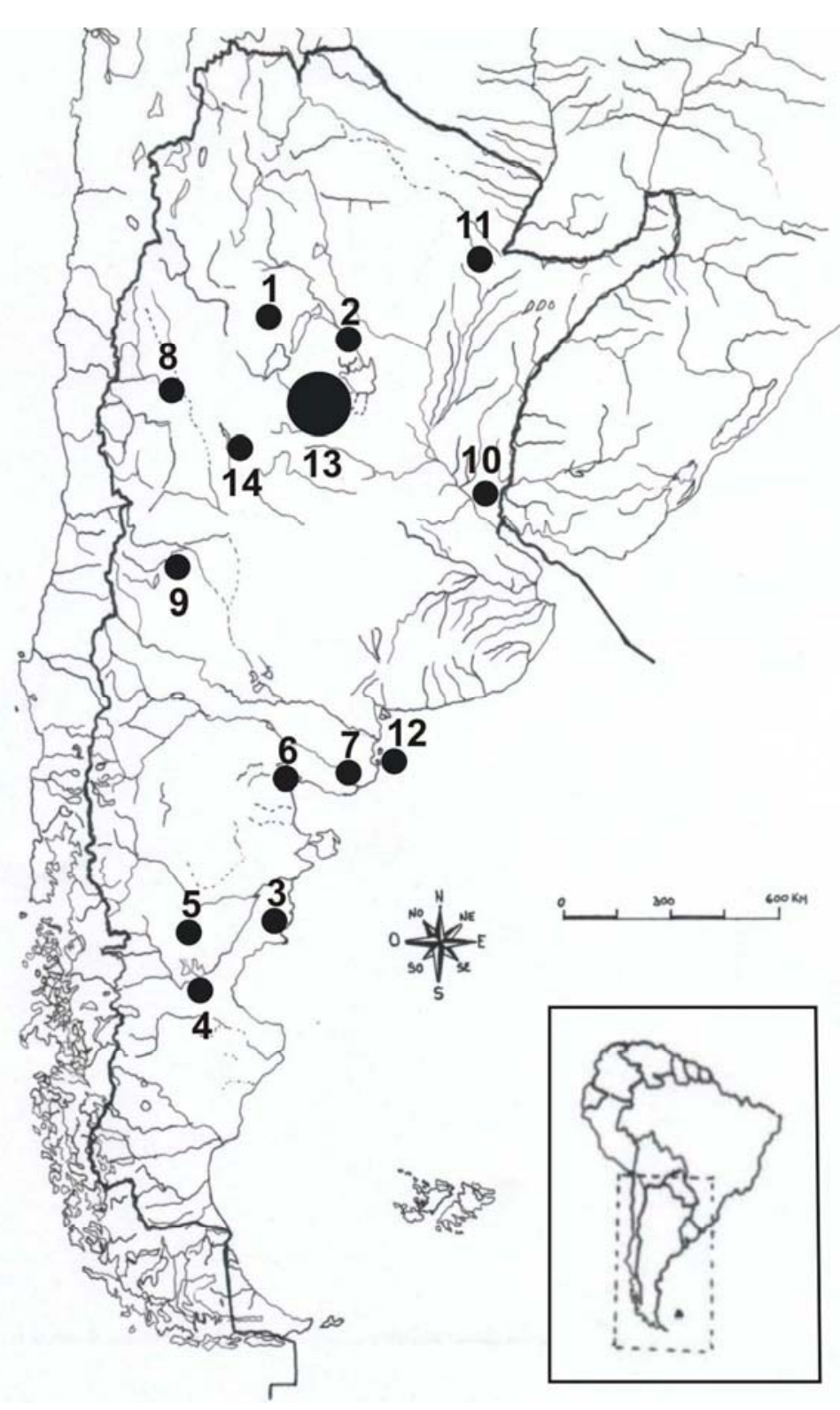

Figura 2. Mapa de la Argentina señalando la ubicación de las 14 muestras incluidas en este análisis: 1) CAT, 2) SGO, 3) NEC, 4) SOC, 5) $\mathrm{CHU}, 6$ ) SAO, 7) RNV, 8) SJU, 9) MZA, 10) ER, 11) CHA, 12) SBI, 13) CBA, 14) SL. El mayor diámetro para CBA denota una mayor dispersión geográfica de los sitios usados en este trabajo. corresponden a cazadores- recolectores terrestres, etnográficamente documentados como Tehuelches. En función de la deformación craneal artificial y de los contextos de inhumación, las muestras de RNV se ubican entre el Holoceno medio (3500-2500 A.P.) y Tardío (2500-400 A.P.) (Bormida 1950, 19531954), mientras SBI ha sido asignado al Holoceno tardío final (Madrid y Barrientos 2000). Las muestras de la Patagonia central proceden de diferentes regiones de la provincia de Chubut, y fueron agrupadas en función de su cercanía geográfica: Valle del Río Chubut y costa central (NEC), Lago Colhue Huapi y Río Mayo (SOC) y muestras de la provincia de Chubut, sin procedencia definida (CHU). Las muestras de la Patagonia central se ubican en el Holoceno tardío (1500-400 A. P), de acuerdo a la deformación craneal artificial. Las muestras de las regiones chaco-santiagueña (SGO -provincia de Santiago del Estero-), Noroeste (CAT provincia de Catamarca-), y porción Norte del Centro-Oeste del país (SJU -provincia de San Juan-) corresponden a poblaciones sedentarias que desarrollaron prácticas agrícolas en el Holoceno tardío. Las muestras de la porción Sur del Centro-Oeste (MZA sur de la provincia de Mendoza-) fueron datadas en el Holoceno tardío y corresponden a grupos humanos que desarrollaron economías mixtas, basadas principalmente en la caza y la recolección, y en menor medida, la agricultura del maíz en períodos tardíos (Novellino et al. 2004). Finalmente, las muestras del Noreste del país (CHA -provincia de Chaco-) y del Noreste de la región pampeana (ER -Delta del río Paraná, provincia de Entre Ríos-), representan poblaciones con una economía mixta, basada en la agricultura, la caza, la recolección y la pesca, que pueden ser asignadas al Holoceno tardío.

\section{Métodos analíticos}

Para controlar la variación morfológica debida a diferencias sexuales, solamente se 
trabajó con individuos masculinos. Las determinaciones sexuales fueron realizadas siguiendo los métodos propuestos por Buikstra y Ubelaker (1994). El registro de las medidas lineales se realizó a partir del set propuesto por Buikstra y Ubelaker (1994), definidas previamente por Martín y Saller (1957). Las variables craneales utilizadas fueron diámetro bicigomático (3.zy-zy), altura facial superior (10.n-pr), anchura frontal mínima (11.ft-ft), anchura facial superior (12.fmt-fmt), altura nasal (13.nns), anchura nasal (14.al-al), anchura orbital (15.d-ec), altura orbital (16.obh), anchura biorbital (17.ekb) y anchura interorbital (18.d-d) (Figura 3). Los números entre paréntesis y letras indican la codificación otorgada por Buikstra y Ubelaker (1994).

\section{Análisis estadístico}

Para el análisis de las relaciones biológicas interpoblacionales se realizará un análisis de componentes principales (PCA), que puede ser definido como un método de ordenación que permite reducir el número de dimensiones a través de las cuales pueden ser visualizadas las relaciones entre las muestras. Si se parte de un espacio multidimensional donde cada dimensión represente un carácter, los métodos de ordenación reducen el número de dimensiones a través de los cuales puedan ser visualizadas las relaciones entre las muestras. Cuanto más cerca se encuentren entre sí dos muestras proyectadas en el espacio determinado por estas variables, más estrechamente relacionadas deben considerarse. PCA permite representar, según un modelo lineal, un conjunto numeroso de caracteres mediante un número reducido de variables hipotéticas, precisamente, los componentes principales. Los mismos no están correlacionados entre sí, y por lo tanto, se interpretan independientemente unos de otros. Cada componente contiene una parte de la variabilidad total de los caracteres. El primero es el que contiene la mayor variabilidad. De la variabilidad restante, el segundo componente es el que incluye más información, y así se continúa hasta que toda la variabilidad ha sido distribuida diferencialmente entre los componentes. Cada componente contiene información de todos los caracteres pero en diferentes proporciones.

\section{Barreras genéticas}

Como se mencionó anteriormente, un tema que por lo general queda sin resolver es el de los límites geográficos de la variabilidad biológica o molecular observada entre poblaciones. Para tal fin, se aplicó un método geométrico computacional basado en el algoritmo de máxima diferencia de Monmonier (1973), el cual provee no solamente la ubicación sino la direccionalidad de las barreras, y muestra donde los patrones geométricos de dos o más variables son similares. Este algoritmo puede ser usado para

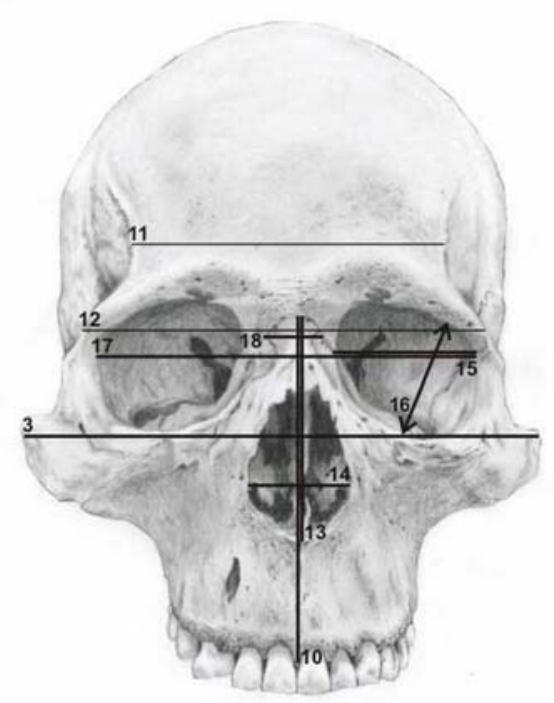

Figura 3. Cráneo en norma frontal señalando las 10 variables lineales consideradas en el presente estudio. Referencias: 3: diámetro bicigomático (3.zy-zy), 10: altura facial superior (10.n-pr), 11: anchura frontal mínima (11.ft-ft), 12: anchura facial superior (12.fmt-fmt), 13: altura nasal (13.nns), 14: anchura nasal (14.al-al), 15: anchura.

identificar fronteras o barreras («boundaries») donde las diferencias entre pares de poblaciones son mayores, dado que maximiza las diferencias genéticas entre poblaciones (Petit et al. 1999; Manni y Barrai 2001). Por este método se obtiene una triangulación de Delauney (Brassel y Reif 1979) uniendo pares de poblaciones localizadas en un mapa, de acuerdo a datos de longitud y latitud. En este estudio, las 14 poblaciones fueron conectadas por 27 límites («edges»). Cada límite fue asociado con un valor de distancia genética. La primera barrera se origina entre pares de poblaciones que presentan el mayor valor de distancia genética. Esta barrera luego se extiende sobre uno de sus límites adyacentes, el cual se asocia con el mayor valor siguiente de distancia genética. Este procedimiento se repite hasta que las fronteras genéticas llegan al límite de la red. En síntesis, la metodología puede resumirse en los siguientes pasos (Manel et al. 2003):

1 - Las poblaciones son ubicadas en un mapa de acuerdo a su ubicación geográfica relativa

2- La triangulación de Delauney se utiliza para conectar las posiciones geográficas adyacentes de las poblaciones en un mapa, resultando una red que conecta todas las muestras.

3- Se calcula las distancias genéticas entre las poblaciones vecinas y se las asocia con cada límite (correspondiente a la muestra vecina) en la red.

4- Se aplica el algoritmo de máxima diferencia de Monmonier para identificar barreras:

- El límite de la red asociado a la mayor distancia genética obtenida es seleccionado y utilizado para iniciar la primer barrera, perpendicular a los límites de la red.

- El límite directamente adyacente a la barrera iniciada con la mayor distancia genética es seleccionado para continuar la barrera. 


\begin{tabular}{lcc}
\hline Variables & $\mathrm{PC1}$ & $\mathrm{PC2}$ \\
\hline 3. Diámetro bicigomático & 0.8966 & 0.0596 \\
10. Altura facial superior & 0.5573 & 0.7738 \\
11. Anchura frontal mínima & 0.5472 & -0.6614 \\
12. Anchura facial superior & 0.8259 & -0.3464 \\
13. Altura nasal & 0.3122 & 0.8364 \\
14. Anchura nasal & 0.4988 & 0.6959 \\
15. Anchura orbital & 0.2274 & -0.2552 \\
16. Altura orbital & 0.3195 & 0.5386 \\
17. Anchura biorbital & 0.7640 & -0.4405 \\
18. Anchura interorbital & 0.8468 & -0.2624 \\
\hline
\end{tabular}

Tabla 3. Análisis de componentes principales. Correlación de variables en cada componente. Primeros dos eigenvectores.

- Los dos pasos previos son repetidos hasta que esta barrera se topa con otra o llega a los límites del área bajo estudio.

Se utilizó el programa Barrier versión 2.2 (Manni et al. 2004) para aplicar el algoritmo de máxima diferencia de Monmonier. Se aplicó este algoritmo a partir del cálculo de la distancia euclidiana cuadrada calculada con el programa NT-SYS versión 1.5 (Rolhf 1989).

\section{Resultados}

\section{Relaciones biológicas entre las muestras}

El análisis de componentes principales (Tabla 3, Figura 4) muestra que las variables que miden el ancho facial (3.zy-zy, 18.d-d, 12.fmt-fmt, 11 .ft-ft y 17.ekb) son las que presentan mayor peso en el primer componente principal (38.96\% de la variación total), con valores positivos. En el segundo componente (29,6\% de la variación), las variables con el mayor peso positivo son las que miden la altura y el ancho facial y nasal (13 nns, 10.n-pr y 14.al-al), mientras que el ancho frontal mínimo (11.ft-ft) tiene un alto peso negativo. Tres agrupamientos pueden ser observados en la Figura 3: poblaciones del Noroeste y CentroOeste del país se ubican en el cuadrante inferior izquierdo, poblaciones de la región chaco-santiagueña y provincia de San Luis ocupan el cuadrante inferior derecho, y el resto de poblaciones de Patagonia, Noreste de la región pampeana, y centro del país se posicionan en los cuadrantes superiores. La muestra CBA se ubica cerca de las poblaciones del Noreste y centro de Patagonia, más específicamente SBI y NEC.

Regiones geográficas con marcado cambio genético: algoritmo de Monmonier

La primer barrera (denominada A en la Figura 5) inicia en el límite entre la muestra de la región chacosantiagueña (SCO) y del Noreste argentino, continuando hacia el sury generando otra barrera con las muestras del Noreste de la región pampeana, región austral de las Sierras Pampeanas o centro del país y norte de la región Centro-Oeste. Es decir, la mayor diferenciación genética se daría entre las poblaciones del Noroeste argentino (CAT y SGO) y el resto de las poblaciones analizadas. La segunda barrera (denominada B) separa a las muestras del Noreste argentino (CHA) y del Noreste de la región pampeana (ER) de las muestras de la región austral de las Sierras Pampeanas (CBA, SL) y el conjunto de muestras patagónicas. La tercera barrera

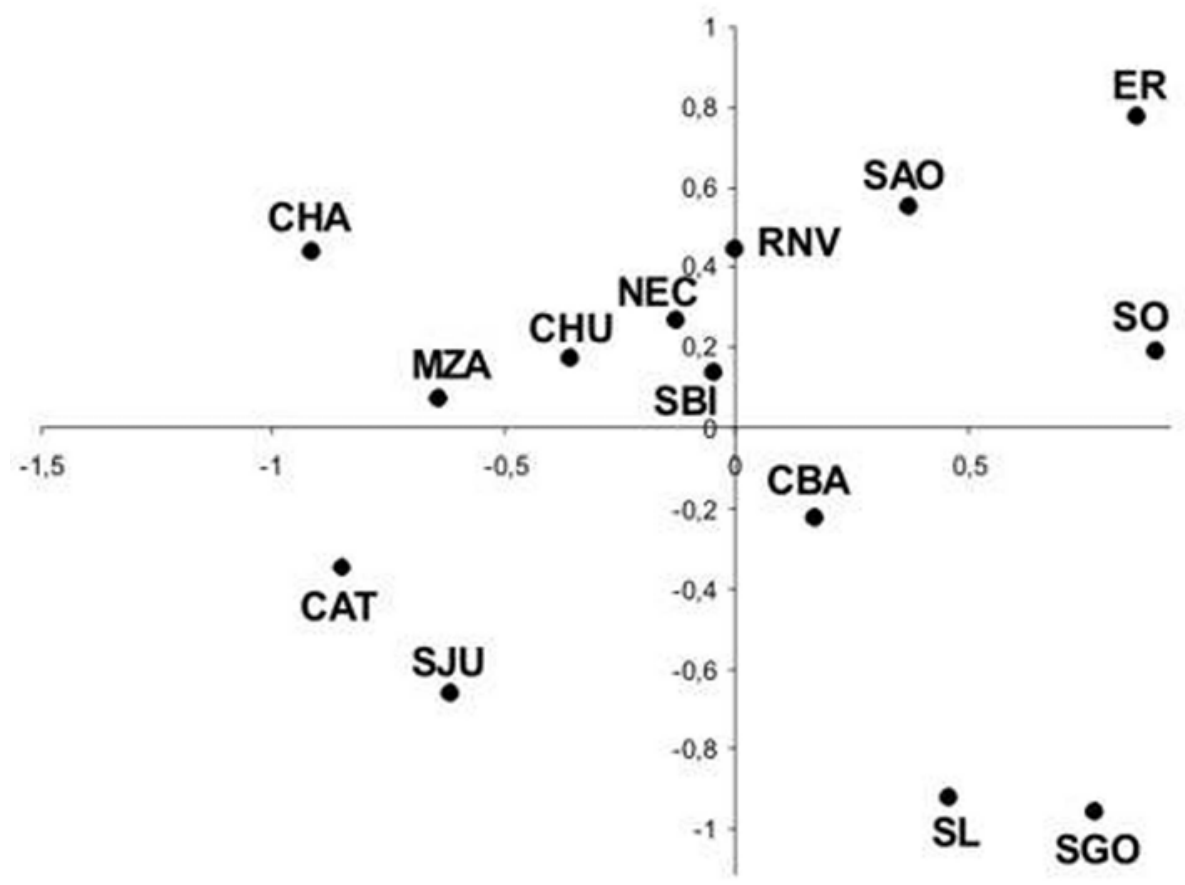

Figura 4. Análisis de componentes principales 


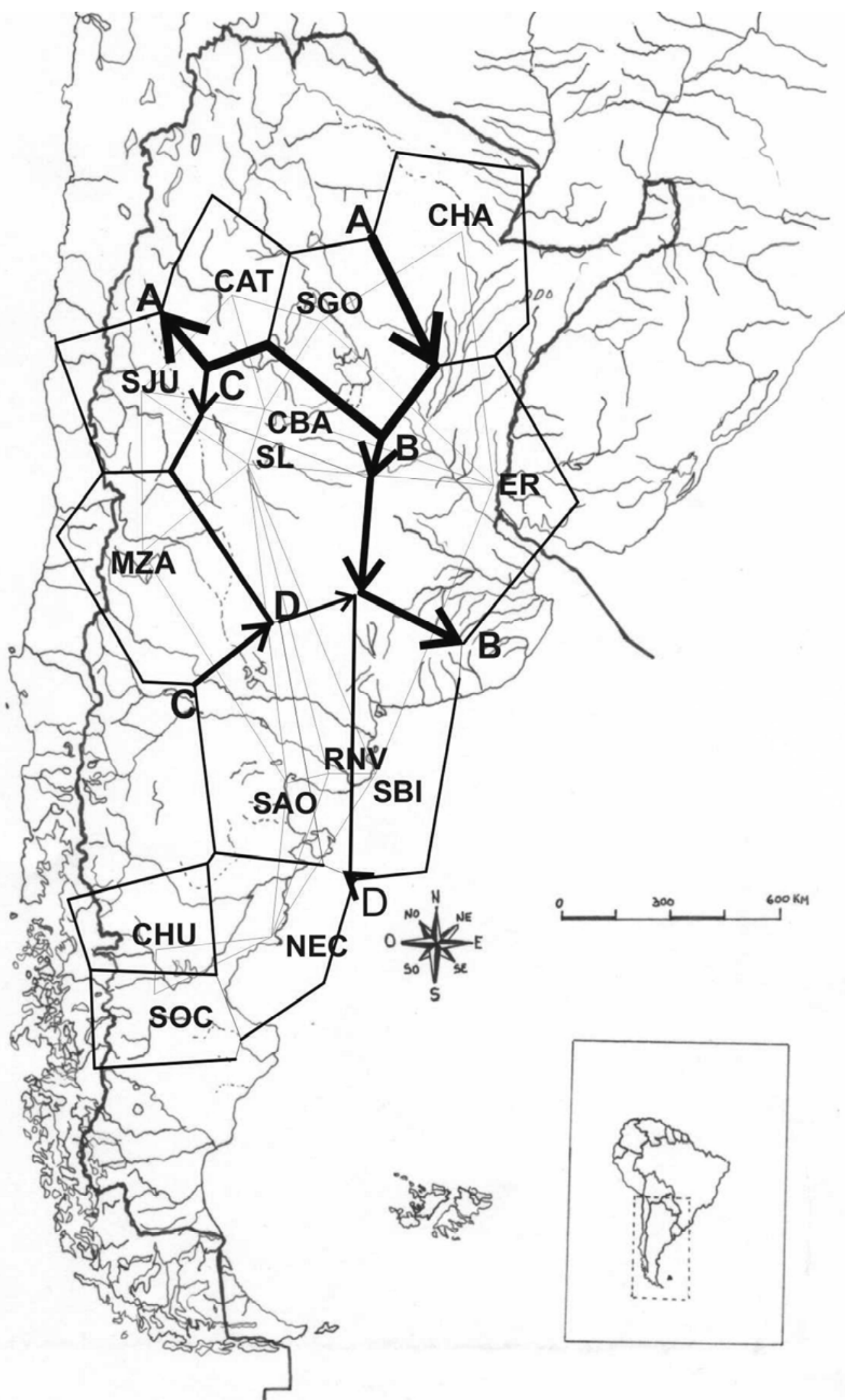

Figura 5. Barreras genéticas detectadas a partir del método de Monmonier (el grosor en los límites de cada barrera es proporcional al número de veces que fue incluido en el cómputo de las barreras)

(denominada C) comenzaría aislando las poblaciones del Centro-Oeste del país de las Noroeste, región austral de las Sierras Pampeanas (CBA, SL) y el resto de las poblaciones patagónicas. La unidad del conjunto que conforman las muestras de la región austral de las Sierras
Pampeanas (CBA, SL) y las poblaciones del Noreste y centro patagónico se rompe recién a partir de la cuarta barrera, lo cual demuestra la elevada similitud biológica entre estas poblaciones. Estos resultados sugieren que las fronteras de mayor diferenciación genética entre las poblaciones 
consideradas seguiría una dirección NoresteSudoeste, dividiendo a las poblaciones del Noroeste, Noreste y Centro-Oeste argentino de aquellas que ocuparon el centro del país y la Patagonia.

\section{Discusión}

El análisis de componentes principales muestra que las poblaciones de la región austral de las Sierras Pampeanas comparten una morfología craneofacial similar con poblaciones del Centro y Noreste de Patagonia (muestras de noreste de Chubut, San Blas e Isla Gama). Las variables que explican esta diferenciación son aquellas que miden la anchura facial (anchura bicigomática, facial superior, frontal mínima y biorbital).

Por otra parte, los resultados obtenidos a partir del algoritmo de Monmonier muestran: a) las poblaciones del Noroeste argentino y de la región chaco-santiagueña claramente diferenciadas del resto, principalmente de las muestras procedentes del centro del país, discontinuidad que resalta aún más considerando la cercanía geográfica entre estas poblaciones y la inexistencia de barreras geográficas que pudieran estar limitando la circulación de personas en estos ambientes, b) una relativa homogeneidad entre la mayoría de las muestras patagónicas (con excepción de la muestra de la península de San Blas e Isla Gama, la cual evidencia mayor similitud con la población del noreste de la región pampeana) lo cual indicaría un elevado flujo génico entre ellas y c) la elevada similitud morfológica entre las poblaciones de la región austral de las Sierras Pampeanas y las patagónicas.

Con respecto a la relación entre variabilidad morfológica craneofacial y temporalidad de las muestras, estudios previos basados en morfometría geométrica han señalado diferencias morfológicas importantes entre muestras de diversos períodos temporales (i.e. Holoceno Medio, Holoceno tardío inicial, Holoceno tardío final) tanto a escala regional (i.e. Sudeste de la región pampeana) como supra regional (Pérez 2006). Pérez (2006) sugiere que las diferencias a escala regional pueden deberse a una reducción demográfica o a un evento de extinción local; esta última hipótesis se vería reforzada por la similitud biológica entre muestras del Sudeste de la región pampeana, Noreste de Patagonia (desembocadura de Río Negro) y Noreste de la región pampeana. En nuestro trabajo, los patrones de variabilidad craneofacial antes mencionados se han definido a partir de muestras que abarcan una escala temporal relativamente amplia: en la mayoría de los casos se está trabajando con muestras cuya cronología las ubica en los últimos 2000 años; particularmente, en el caso de dos muestras (CBA y RNV) esta escala se profundiza, abarcando 4000 años. De allí, que se considere fundamental en futuros estudios refinar la escala temporal de análisis a partir de una mayor información radiocarbónica de las muestras. Un ajustado control cronológico permitirá confirmar el patrón de variación observado o identificar nuevas barreras genéticas en períodos temporales más acotados, y de esa forma, sumar elementos que posibiliten una mayor comprensión de la compleja historia biológica de estas poblaciones.

Los cambios en la morfología craneal deben ser explicados atendiendo a una serie de factores, entre ellos, la influencia de fuerzas evolutivas tanto direccionales -migración, selección- como aleatorias -efecto fundador, deriva génica- pero también ambientales de tipo adaptativos -relación tamaño-peso con la latitud; pigmentación- o plásticos -i.e. deformación craneal- (González-José 2003), así como mecanismos estocásticos. Entre los factores ambientales que pueden influir en la morfología craneal también pueden mencionarse las diversas estrategias de subsistencia económicas desarrolladas por los grupos humanos (Sardi et al. 2006). En este sentido, si consideramos el estilo de vida de estas poblaciones en conjunto con la información obtenida a partir de las barreras genéticas, es sugerente observar que las mayores diferencias morfológicas se observan entre poblaciones que han adoptado la agricultura en momentos tardíos (CAT, SGO, SJU) y aquellas que desarrollaron a) economías mixtas, basadas principalmente en la caza y la recolección, incluyendo en algunos casos la pesca (CHA, ER) o ciertos cultígenos (CBA, MZA) a la dieta, o b) estrategias puramente cazadoras-recolectoras como las poblaciones patagónicas (SBI, NEC, RNV, SAO, NEC, SOC, CHU). Estudios recientes sobre componentes funcionales del cráneo (Sardi et al. 2006) realizados en muestras arqueológicas en las subáreas Norte (San Juan y Norte de Mendoza) y Sur (Sur de Mendoza) de la región Centro-Oeste del país han puesto de manifiesto la influencia de la dieta sobre la morfología craneofacial de poblaciones cazadoras-recolectoras y agrícolas. Las poblaciones que tienen una dieta basada principalmente en la producción de alimentos presentan cambios localizados en componentes funcionales específicos; más precisamente, se observa una reducción en el tamaño de los componentes masticatorios y posteroneuronal del cráneo en poblaciones agrícolas en comparación con grupos cuya estrategia de subsistencia se basa en la caza y la recolección. Este patrón puede estar relacionado con factores localizados -reducción del stress masticatorio- y sistémicos - menor calidad nutricional debido al menor consumo de proteínas en dietas basadas en cultivos, así como disminución de la hormona de crecimiento debido a un mayor sedentarismo de estas poblaciones-. Es interesante pensar que nuestros resultados sobre barreras o límites genéticos entre poblaciones no solamente están poniendo de manifiesto las diferentes trayectorias biológicas de las poblaciones -en función del mayor o menor flujo génico ocurrido entre ellas- sino también diferencias debidas al 
desarrollo de diversas estrategias de subsistencia, particularmente, cuestiones vinculadas a las estrategias de explotación y patrones de consumo de recursos alimenticios.

La semejanza entre las muestras de la región austral de las Sierras Pampeanas con las poblaciones patagónicas sugiere un origen común o un significativo flujo génico entre estas poblaciones. Estos resultados concuerdan con estudios previos basados en medidas lineales que postulan semejanza biológica entre poblaciones humanas que habitaron la región central del país y Patagonia (Colantonio y Marcellino 2000; Marcellino 1992; Torres Mazzuchi 1975), y con nuestros propios resultados, basados en rasgos epigenéticos craneales (Fabra et al. 2005), medidas lineales (Fabra 2008a, 2008b, Fabra et al. 2007) y datos moleculares (García y Demarchi 2006). Al respecto, estos autores han encontrado elevada frecuencia de haplogrupos mitocondriales $C$ y $D$ en poblaciones «criollas» que habitan diferentes localidades de la provincia de Córdoba. La distribución de los linajes maternos amerindios de la muestra de Córdoba es similar a la que se encuentra en poblaciones nativas de Patagonia y Tierra del Fuego. De esta forma, los resultados obtenidos a partir del estudio de la morfología craneofacial así como marcadores moleculares del ADN mitocondrial refuerzan nuestros supuestos acerca de la semejanza biológica de estas poblaciones debida al flujo génico o a un origen común entre estas poblaciones.

\section{Córdoba, 31 de Agosto de 2008}

\section{Agradecimientos}

Esta investigación se desarrolló en el marco del proyecto «Historia de las poblaciones prehispánicas del actual territorio de la Provincia de Córdoba: evidencias bioantropológicas y modelos arqueológicos», dirigido por el Dr. Darío Demarchi y codirigido por el Dr. Andrés Laguens (FONCyT PICT 2003-151 87-y SECyT -Universidad Nacional de Córdoba-). Un especial agradecimiento a aquellos museos que permitieron el acceso a las colecciones humanas bajo su cuidado, particularmente a Mirta Bonnin (Museo de Antropología, F.F.y H., Universidad Nacional de Córdoba), Inés Baffi (Museo Etnográfico «Juan Bautista Ambrosetti», Universidad de Buenos Aires), Héctor Pucciarelli, (División Antropología, Facultad de Ciencias Naturales y Museo, Universidad Nacional de La Plata), Paula Novellino (Museo Municipal de Historia de San Rafael, Mendoza), Museo «Dora Ochoa de Masramón» (San Luis), Museo Histórico Municipal de La Para. (La Para, Córdoba), Museo de la región de Ansenuza «Aníbal Montes» (Miramar, Córdoba), Museo de Marull (Marull, Córdoba), Museo Arqueológico provincial «Aníbal Montes» (Río Segundo, Córdoba), Museo Municipal «Capitán Juan de Zevallos» (Valle Hermoso, Córdoba), Museo Sanavirón (San José de la Dormida, Córdoba), Museo Dr. Dalmacio Vélez Sársfield (Amboy), Museo Arqueológico e Histórico
Regional «Florentino Ameghino» (Río Tercero), Museo Rocsen (Nono), Museo Comechigón (Mina Clavero), Museo Regional de Villa Dolores (Villa Dolores y colección privada de familia Verde (Cerro Colorado, Córdoba) y familia Gallo (Tilisarao, San Luis). La realización de los mapas e ilustraciones que acompañan a este trabajo estuvo a cargo de Paola Sebal. Finalmente, se agradecen los comentarios realizados por la Dra. Paula González, los cuales sin duda ayudaron a mejorar sensiblemente este trabajo.

\section{Bibliografía}

Barrientos, G., H. Pucciarelli, G. Politis, S. I. Pérez, M. Sardi. 2003. The Craniofacial Morphology of the Early- to Middle- Holocene Human Populations from the Pampean Region, Argentina: Getting a New Insight into the Morphological Variability of Early Americans. L. Miotti, M. Salemme y N. Flegenheimer (Eds.) Where the South Winds Blow. Ancient Evidence for Paleo South Americans, 6976. College Station: (CSFA) and Texas A\&M University Press.

Bernal, V., S. I. Pérez, P. N. González. 2006. Variation and causal factors of craniofacial robusticity in Patagonian hunter-gatherers from Late Holocene. American Journal of Human Biology, 18:748-765.

Bonnin, M., A. Laguens. 2000. Esteros y algarrobales. Las sociedades de las Sierras Centrales y la llanura santiagueña. M. Tarrago (Ed), Nueva Historia Argentina, Tomo I Los pueblos originarios y la conquista, Editorial Sudamericana, 1: 149-186.

Bórmida, M. 1950. Cementerios indígenas prehispánicos en la zona Laguna del Juncal. Anales del Museo Nahuel Huapi, 2:101-108.

Bórmida, M. 1953-1954. Los antiguos Patagones. Estudios de craneología. Runa, 6 (1-2): 55-96.

Buikstra, J. E., D. H. Ubelaker (eds.). 1994. Standards for data collection from human skeletal remains. Arkansas Archaeological Survey Research series, No. 44. Fayetetteville: Arkansas Archaeological Survey.

Brassel, K.E., D. Reif. 1979. A procedure to generate Thiessen polygons. Geogr. Anal, 325:31-36.

Cabana, G., A. D. Merriwether, K. L. Hunley, D. A. Demarchi. 2006. Is the genetic structure of Gran Chaco populations unique? Interregional perspectives on Native South American mitochondrial DNA variation. American Journal of Physical Anthropology 131:108-119. 
Canals Frau, S. 1944. El grupo HuarpeComechingón. Anales del Instituto de Etnografía Americana, 5: 25-53.

Capitanelli, R. G. 1979. Geomorfología. J. B. Vázquez, R. A. Miatello y M.E. Roqué (eds), Geografía física de la provincia de Córdoba, Editorial Boldt, Bs.As, 213-296.

Cocilovo, J. A. 1984. Una nueva aproximación al conocimiento de la población prehistórica de la provincia de Córdoba, Comechingonia, 2 (3):85-104.

Cocilovo, J. A., J.A. Di Rienzo J. A. 1984-1985. Un modelo biológico para el estudio del poblamiento prehispánico del territorio argentino. Correlación fenético-espacial. Relaciones de la SociedadArgentina de Antropología, Buenos Aires, 16: 119-135.

Cocilovo, J. A, W. A. Neves. 1988-1989. Afinidades biológicas entre las poblaciones prehistóricas del Litoral del Brasil y de Argentina. Primera Aproximación. Relaciones de la Sociedad Argentina de Antropología, $17(2): 31-56$.

Cocilovo, J.A., H. Varela, E. I. Baffi, S. Valdano. 1999. Estructura y composición de la poblacion antigua de la Quebrada de Humahuaca. Análisis Multivariado. Revista Argentina de Antropología Biológica, 2(1): 7-26

Colantonio, S., A. Marcellino. 2000. Vinculaciones morfológicas entre series craneanas aborígenes del Cono Sur americano. Tendencias actuales de investigación en la antropología física española, Secretariado de Publicaciones, Universidad de León, España, 219-232.

Dejean, C. B., B. Crouau-Roy, A. S. Goicoechea, S.A Avena, F. R. Carnese. 2004. Genetic variability in Amerindian Populations of Northern Argentina. Genetic and Molecular Biology 27:489-495.

Demarchi, D. A. 2000. Genetic structure of native Andean populations from Argentina inhabiting in different altitudes, Human Biology, 73:575-582

Demarchi, D. A, G. M. Panzetta-Dutari, C. C. Motrán, M. D. L. de Basualdo, A. J. Marcellino. 2001. Mitochondrial DNA haplogroups in Amerindian populations from the Gran Chaco, American Journal of Physical Anthropology, 1 15:199-203.

Demarchi, D. A., F. M. Salzano, M. E. Altuna, M. Fiegenbaum, K. Hill, A. M. Hurtado, L. T. Tsunetto, M. L. Petzl-Erler, M. H. Hutz. 2005. ApoE polymorphisms distribution among Native Americans and related populations, Annals of Human Biology, 32 (3): 351-365.

Demarchi, D. A., M. Fabra, A. García. 2007. Poblamiento inicial de las Sierras Centrales de Argentina: nuevas evidencias morfológicas y moleculares. Memorias del III Simposio Internacional «El Hombre temprano en América» (DAF/INAH, México). En prensa.

Dipierri, J. E., E. Alfaro, V. L. Martinez Marignac, G. Baillet, C. M. Bravi, S. Cejas, N. O. Bianchi. 1998. Paternal directional mating in two Amerindian subpopulations located at different altitude in the northwest of Argentina. Human Biology, 70:1001-1010.

Dipierri, J. E., E. Alfaro, J. A. Pena, J. Constans, J. M. Dugoujon. 2000. Km inmunoglobulin allotypes and other serum genetic markers (hp, gc, pi and tf) among South American populations living at different altitudes (Jujuy Province, Argentina): admixture estimates. Human Biology 72(2):305319.

Fabra, M. 2008a. El poblamiento humano del actual territorio de Córdoba: una interpretación a partir de modelos arqueológicos y evidencias bioantropológicas. Serie Tesis de Postgrado. Secretaría de Ciencia y Tecnología, Facultad de Filosofía y Humanidades, Universidad Nacional de Córdoba. En prensa

Fabra, M. 2008b. Relaciones biológicas craneales entre poblaciones prehispánicas de la región central del territorio argentino y otras del Cono Sur sudamericano. Revista Anales de Arqueología y Etnología de la Facultad de Filosofía y Letras de la Universidad Nacional de Cuyo. En prensa

Fabra, M., A. G. Laguens, D. A. Demarchi. 2005. Análisis intra e inter poblacional de rasgos craneanos no métricos en aborígenes prehispánicos del actual territorio de Córdoba. Revista Argentina de Antropología Biológica, 7 (2):47-65.

Fabra, M., A. G. Laguens, D. A. Demarchi. 2007. Human Colonization of the Central Territory of Argentina: Design Matrix Models and Craniometric Evidence. American Journal of Physical Anthropology, 133(4): 1060-1066.

García, A, D. Demarchi. 2006. Linajes parentales amerindios en poblaciones del Norte de Córdoba. Revista Argentina de Antropología Biológica, 8(1): 5771

Goicoechea, A. S., F. R. Carnese, C. B. Dejean, S. A. Avena, T. A. Weimer, M. H. Franco, S. Callegari-Jacques, A. Estalote, M. L. Simoes, M. Palatnik, P. Salomeni, F. M. Salzano. 2001. Genetic relationships between Amerindian populations of Argentina. American Journal of Physical Anthropology, 115:133-143.

González, A. R. 1944. Algunas observaciones sobre los caracteres antropológicos de los primitivos habitantes de Córdoba. Publicaciones del Instituto de Arqueología, Lingüística y Folklore, Universidad Nacional de Córdoba, 1-8. 
González-José, R. 2003. «El poblamiento de la Patagonia. Análisis de la variación craneofacial en el contexto del poblamiento americano». Tesis Doctoral. Universidad de Barcelona, España, 363 páginas. Inédita.

González-José, R., S. Dahinten, M. Hernández. 2001a. The settlement of Patagonia: a matrix correlation study. Human Biology 73: 233-248.

González-José, R., S. Dahinten, M. Hernández. $2001 \mathrm{~b}$. Craniometric variation and the settlement of the Americas: testing hypothesis by means or $\mathrm{R}$-matrix and matrix correlation analysis. American Journal of Physical Anthropology 116 : 154-165.

González-José, R., A. González-Martin, M. Hernández, H. Pucciarelli, M. Sardi, A. Rosales, S. Van Der Molen. 2003. Craniometric evidence for Paleoamerican survival in Baja California. Nature, 425: 62-65.

Hernández, M., C. Lalueza Fox, C. García Moro. 1997. Fueguian cranial morphology: adaptation to a cold, harsh environment. American Journal of Physical Anthropology 103:103-17.

Laguens, A. 1999. Arqueología del contacto hispano-indígena. Un estudio de cambios y continuidades en las Sierras Centrales de Argentina. BAR International Series 801, Oxford.

Laguens, A., D. A. Demarchi, M. Fabra. 2007a. Un estudio arqueológico y bioantropológico de la colonización humana en el sector sur de las Sierras Pampeanas. Debates actuales en Arqueología y Etnohistoria. Publicación de las V y VI Jornadas de Investigadores en Arqueología y Etnohistoria del Centro-Oeste del país. Foro Pueblos originarios-arqueólogos, Universidad Nacional de Río Cuarto, 119-134.

Laguens, A., G. R. Cattáneo, E. Pautassi, G. Sario. 2007b. Poblamiento humano temprano en las Sierras de San Luis: Estancia La Suiza. VII Jornadas de investigadores en Arqueología y Etnohistoria del Centro Oeste del país, Laboratorio de Arqueología y Etnohistoria Departamento de Historia, Facultad de Ciencias Humanas Universidad Nacional de Río Cuarto. En prensa.

Laguens, A, E. Pautassi, G. Sario, G. R. Cattáneo. 2007c. ELS 1, a Fishtail Projectil Point from Central Argentina. Current Research in the Pleistocene 24: 55-57.

Lahr, M. M. 1995. Patterns of modern human diversification: implication for Amerindian origins. Yearbook American Journal of Physical Anthropology 38:163-198.
Lalueza Fox, C., M. Hernández, C. García Moro. 1996. Craniometric analysis in groups from Tierra del Fuego/Patagonia and the peopling of the southern extreme of the Americas. Human Evolution 11 (3-4):217-224.

Lalueza Fox, C., M. Hernández, C. García Moro. 1997. La morfología facial de las poblaciones fueguinas: ¿reflejo de una adaptación al frio? An Inst Patagon Ser Cs Hu, 25: 45-58.

Madrid, P., G. Barrientos. 2000. La estructura del registro arqueológico del sitio Laguna Tres Reyes 1 (Provincia de Buenos Aires): nuevos datos para la interpretación del poblamiento humano del sudeste de la región pampeana a inicios del Holoceno tardío. Relaciones de la Sociedad Argentina de Antropología, 25: 179-206.

Manel, S., M.K Schwartz., G. Luikart, P. Taberlet, 2003. Landscape genetics: combining landscape ecology and population genetics. Trends in Ecology and Evolution, Vol.1 8 (4):1 89-197.

Manni, F., Barrai, I. 2001. Genetic structure and linguistic boundaries in Italy: amicroregional approach. Human Biology, 73, 335-347.

Manni, F., E. Guerard, E. Heyer. 2004. Geographic patterns of (genetic, morphologic, linguistic) variation: how barriers can be detected by «Monmonier's algorithm». Human Biology, 76 (2): 173-190.

Marcellino, A. J. 1992. Síntesis historiográfica de los estudios antropológicos en la provincia de Córdoba. Cuadernos de Historia, Junta Provincial de Historia de Córdoba, 11-47.

Marcellino, A. J, S. Colantonio. 1993. Relaciones morfológicas de los aborígenes prehispánicos del territorio Argentino. VII: la región serrana de Córdoba. Separata de la Revista Nro. 15 de la Junta provincial de Historia de Córdoba, Córdoba, $113-$ 135.

Marcellino, A. J, S. Colantonio. 2000. Los cráneos aborígenes más antiguos de Argentina: un ensayo clasificatorio. Tendencias actuales de investigación en Antropología Física Española, Secretariado de Publicaciones, Universidad de León, España, 205-218.

Martin, R, K. Saller. 1957. Lehrbuch der anthropologie, I. Ed. Gustave Fisher, Stuttgart, Alemania.

Méndez, S., S. Salceda. 1995. Metric and non metric variants in prehistoric populations of Argentina. Rivista di Antropologia (Roma), 73: 145-158. 
Monmonier, M. 1973. Maximum-difference barriers: an alternative numerical regionalization method. Geog. Anal. 3, 245-261.

Novellino, P., A. Gil, G. Neme, V. Duran. 2004. El consumo del maíz en el Holoceno Tardío del Oeste Argentino: isótopos estables y caries. Revista Española de Antropología Americana, 34:85-1 10.

Outes, F. 1911. Los tiempos prehistóricos y protohistóricos en la provincia de Córdoba. Separata de la Revista del Museo de La Plata, Buenos Aires, 7 (4): 261-374.

Pérez, S. I. 2006. «El poblamiento holocénico del sudeste de la región pampeana: un estudio de morfometría geométrica craneofacial», Tesis de doctorado, Universidad Nacional de La Plata, 131 páginas, Inédita.

Pérez, S. I., V. Bernal, P. N. González. 2007. Morphological differentiation of aboriginal human populations from Tierra del Fuego (Patagonia): implications for South american peopling. American Journal of Physical Anthropology, 133: 1067-1079.

Petit, E., L. Excoffier, F. Mayer. 1999. No evidence of bottleneck in the postglacial recolonization of Europe by the noctule bat (Nyctalus noctula). Evolution, 53 (4): 1247-1258.

Rohlf, F. J. 1989. NTSYS-PC numerical taxonomy and multivariate analysis system. New York, Exeter Publishing.
Rothammer, F., J. A Cocilovo, S. Quevedo. 1984. El poblamiento temprano en Sudamérica, Chungará, 13: 99-108

Rothammer, F., M. Acuña, E. Llop. 1988-1989. La población de Sudamérica: nuevos aportes basados en el análisis de cráneos arcaicos y frecuencias génicas de aborígenes contemporáneos. Relaciones de la Sociedad Argentina de Antropología, 17 (2): 19-30.

Sardi, M., F. Ramírez Rozzi, R. González-José, H. Pucciarelli. 2005. South American craniofacial morphology: diversity and implications for amerindian evolution. American Journal of Physical Anthropology, 128:747-756.

Sardi, M., P. Novellino, H. Pucciarelli. 2006. Craniofacial morphology in the Argentine Center.West: consequences of the transition to food production. American Journal of Physical Anthropology, Vol. 130:333-343.

Serrano, A. 1945. Los Comechingones. Serie aborígenes argentinos, Volumen 1, Instituto de Arqueología, Lingüística y Folklore, Universidad Nacional de Córdoba.

Torres Mazzuchi, M. H. 1975. Estudio antropológico de esqueletos de aborígenes de Río Segundo (Pvcia. de Córdoba, Argentina). Revista del Instituto de Antropología, Universidad Nacional de Tucumán, Facultad de Filosofía y Letras, Tercera Serie, 2:147169. 